Full length article

\title{
RELATIONSHIP BETWEEN METROLOGICAL PARAMETERS AND VECTOR BORNE DENGUE DISEASE USING ONCOLOGICAL FRACTAL TREATMENT
}

\author{
M. Ilyas', S. Abbas', *, A. Ali', S. Hussain² and S. A. Raza 3
}

1.Laboratory for Applied Mathematics and Data Analysis, Mathematical Sciences Research Centre, Federal Urdu University of Arts, Sciences and Technology, Karachi, Pakistan.

2.Department of Mathematics, University of Baltistan, Skardu, Pakistan.

3.Department of Computer Sciences, Federal Urdu University of Arts, Sciences and Technology, Karachi, Pakistan.

\begin{abstract}
Dengue is the most vital arboviral disease in humans, which is occurring in tropical and subtropical areas around the world. Dengue fever is itemized as an urban human disease as it spreads easily to urban environmental/morphological contexts, because of the uneven increase of urban population and infectious diseases as a result of climate change. Dengue epidemic cases related to climatic parameters are helpful to monitor and prevent the transmission of dengue fever. Many studies have focused on describing the clinical aspects of dengue outbreak. We bring out the epidemiological study to investigate the dengue fever development and prediction in the Karachi city. This study described the oncological treatment by statistical analysis and fractal rescaled range $(R / S)$ method of the dengue epidemics from January 2001 to December 2020, based on the urban morphological patterns, and climatic variables including temperature and ENSO respectively. The R/S method in oncologists has been carried in two ways, basic oncological/statistical analysis and Fractal dimension adapt to the study the nature of the subtleties of dengue epidemic data, another showing the dynamics of oncological process. Climate parameters are shown that the fractal dimension value revealed a persistency behavior i.e. time series is an increasing, Fractal analysis also confirmed the anti-persistent behavior of dengue for months of September to November and the normality tests specified the robust indication of the intricacy of data. This study will be useful for future researchers working on epidemiology and urban environmental oncological fields to improve and rectify the urban infectious diseases.
\end{abstract}

KEYWORDS:Dengue Epidemic, Urban human arboviral disease, Urban Morphology, Fractal Dimension, Climatic Parameters

*Corresponding author: (Email: shaheen.abbas@fuuvast.edu.pk, 03333239969)

\section{INTRODUCTION}

The vector-borne dengue disease is the most significant arboviral disease in the world, according to morbidity and mortality, which estimates that fifty (50) to hundred (100) million cases and twelve thousand to twentyfour thousand deaths per year [11, 15]. The vector borne, Aedes aegypti mosquito, live in a handy urban and suburban areas secure to humans, where lavas are usually prepared in water vessel for example barrels, buckets, tires and blossom pots [3, 22]. Overall, global factors around dengue viruses and Ae. Aegypti have been affected by such factors as population growth or development and unplanned areas such as slums as well as unplanned urban morphology with respect to urban climate [31]. Dengue fever is recorded as an urban disease as it facilitates urban contexts because an unprecedented increase in urban population. 
Dengue fever epidemic in southern Asia, including in Pakistan, since 1996 has increased dengue transmission. In recent decades, dengue has become tropical countries in a growing urban health problem [2]. The disease is assumed to be spread mostly as an outcome of in effectible vector and disease surveillance; insufficient human health infrastructure; population growth; unplanned and uncontrolled urban morphological structures and urban climate variation. The association of climate parameters and the dengue fever epidemic in the first decay of the 20th century, in that time, some researchers have tried to connect the relation between these climate parameters and the dengue fever. The climate parameters Precipitation, Temperature and El NiñoSouthern Oscillation index are helping outs is reduced to the time interval for the imitation of the dengue fever virus [33]. In the high temperature mosquito grows to infectious rapidly and high probability to human beings before its impact on the effect of the risk. Climatic revolution and climate factors are being used to serve dengue vector but there are many other factors also co-operating a vital role for the dengue outbreak $[16,17,18]$. The spread of dengue fever and expansion can help connect among urban morphology, climate and public health [39]. The vectorborne dengue diseases (VBDDs) epidemics and spreading in urban population is apparently multi-factorial, about the biological, metrological and social factors for climate change is long since smeared as significant in figure 1.1.

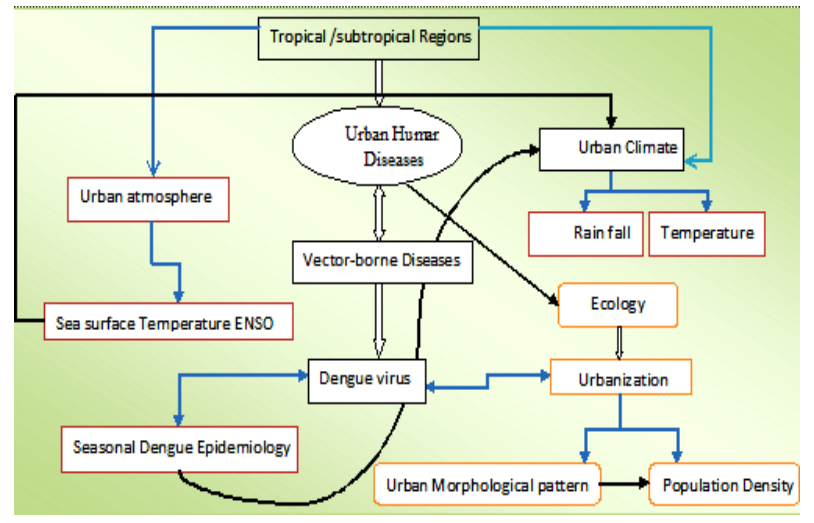

Figure 1.1. Vector Borne dengue disease transmission related to urban morphology and climatic parameters.

Urban morphology is defined in various settle countries that categorize and organizes the distribution of urban functions. In urban planning, it is supported and detect evolving problems and solve urban disorders $[12,33,37$, 39,46 ] as figure 1.1 is also clearly mention the dynamics of the dengue disease is obscured because its transition process depends on urban morphological functions such as urban environmental/ ecological and climate change. In epidemiology, a typical example of infectious dynamic is complex behavior [35]. There are some standard approaches about the distribution of predictor and resultant variables (Weaver and Vasilakis 2009; Gubler et al. 1984; Balmaseda et al. 2005) in the standard modes of data epidemiology. We generally understand that invisible, random noise in epidemiology study, which can be determined and corrected in statistical data. To study these types of systems, the basic mathematical Oncological approaches are to apply fractal theory. However, biological sciences, including epidemiologists, are considered as biological concepts, according to the most valuable values for most biology [13, 21, 22]. The aim of this study are to assess whether or not the fractal method can be used in some field of 
epidemiology, and the practical analysis approach to studying where a revelry view can be rummage-sale and more linear areas where the outmoded viewpoint is correct [19].

This study demonstrates, the use of fractal theory in oncologists are bring out up to now in two methods: by statistical analyzing of the dengue transmission in the influence of urban climate parameters, However, other hand determined the persistency behavior of the time series by using self-affine fractal dimension (rescaled range method). The use of the fractal method range performing the dynamics of the oncological process. The fractal theory is a unified concept of scale dependence and complexity, both of whom think of both biological patterns and practices [20]. The fractal dimension is also a numeric interpreter that determines the morphological structural complexity of the biological elements of its symptoms.

This study is distributed the various parts, in section II, articulate the data description and briefly converse all techniques, section III, obtain the results and detail in discussions and last section IV, conclude all outcomes.

\section{DATA DESCRIPTION AND METHOD OF}

\section{ANALYSIS}

Karachi city is the most populated city of Pakistan and has the most important port and financial center. The population of 23.5 million people is estimated in April 2020 and a population density approximately 6,000 per square kilometers (15,500 per square miles) and the World's third largest city is population [3]. The city of Karachi has faced many problems with uncontrollable urbanization (urban morphogenesis), urban management and planning. Rapid urbanization has caused the increase of built-up coverage with disordered typologies, incomplete infrastructure, and urban-peripheral instability. The city of Karachi is located to the subtropical climate region. Karachi and its suburbs lie in semi-arid Zone. Rainfall in the region is very uncertain and irregular. Karachi has two important seasons; summer and winter, while spring and autumn are very short. During the summer the summer continues for the long run. Karachi has a moderate climate, which occasionally becomes dry, otherwise, intensely humid. The average monthly wind speed during the year varies from 3.2 to 10.7 knot per hours. The hottest months are May, June, September and October. The mean daily maximum temperature ranges between $33.33{ }^{\circ} \mathrm{C}$ to $46.6{ }^{\circ} \mathrm{C}$ lowest temperature recorded being $5^{\circ} \mathrm{C}$. An ENSO index, which has led to increasing the risk of dengue spread. This is a marked effect on the dengue disease when values range from $27^{\circ} \mathrm{C}$ to $28^{\circ} \mathrm{C}$, but a sharp decline when the El Nino is less than $27^{\circ} \mathrm{C}$ [27].

The city of Karachi is distributed in high population city of Sindh province. The size of a city also exhibits certain spatial population density distribution patterns. For the administrative purposes before the year 2000 Karachi are distributed in five districts. In the year 2000 Karachi are divided into subunits [41, 42]. In 2001, five districts of Karachi were found together with a three-level structure to make district of Karachi. The two most local buildings consist of sixteen towns. This changeover greatly disturbed the population distribution pattern of Karachi. We have collected the population census data of subunits (districts and towns) of the Karachi city for the period of 2002 respectively. 
According to our constructed spatial population density map (Figure 1.1) illustrates, the Morphogenesis pattern of the urban density in proportion to urban population of Karachi region according to 2002 census records. The GIS population map of subunits (Districts and Towns) are created from the Visualize ESRI shape files of Karachi City available on the internet. Population data for each district and towns are then encoded and finally joined with the Karachi boundary shape files. For purpose of distance measurement, pivot of each polygon was derived with "feature to point" option in Arc GIS. Dengue fever has one of the most seasonal viral diseases. On the basis of our constructed GIS map (figure 1.1) we have found that the city is facing the morphological structural changing due to unplanned and uncontrolled population of the city. Urban uncontrolled population and urban climate changing is main cause of the seasonal vector-borne viral diseases such as dengue fever, chicken gunia in the duration of 2001 and 2020. More density areas and unplanned and undeveloped slums areas are more effected for dengue epidemic. The tropical vector-borne seasonal disease which have caused of climatic change extreme land surface temperature and El Nino southern oscillation (ENSO) index over recent decades. Due to urbanization, incomplete infrastructure and an unprecedented population of urban morphology has reshaped. In the context of seasonal vectorborne disease such as dengue epidemics, these problems have increased more seriously issue in the influence of dengue epidemics to the approaching of absurd planning and management in the city.

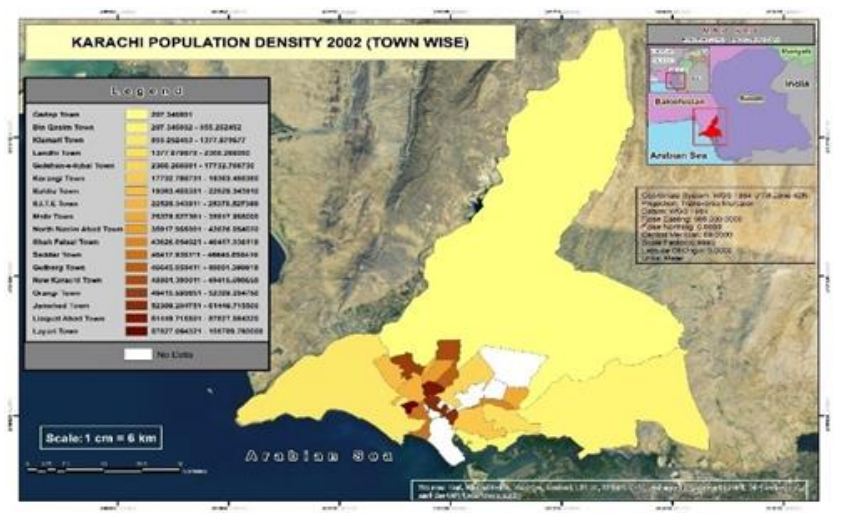

Figure1.2. The Spatial population density map is indicated spatial structure of local government areas of the Karachi city.

Data on monthly dengue counts duration from January 2001 to December 2020 are obtained from the dengue survival cells, climate data are collected from Karachi metrological department, the Karachi city weather station $(23 \circ \mathrm{N}, 68 \circ \mathrm{E})$, which records temperature on an hourly basis. In tropical Pacific, ocean conditions are the main drivers of rotation in the atmosphere and can have significant impact on the global climate. (ENSO) is an important element of the climate system and its variations are necessary to understand the possible changes, which may be due to natural process, such as environmental or orthopedic effects [9]. These data are summarized on mean monthly temperature (from 2001 to 2020). We have also collected the monthly ENSO 3.4 index in the analysis. The monthly ENSO Index (from 2001 to 2020) are obtained from the Global Climate observing system, which is supported by the National Oceanic and Atmospheric Administration

(www.ncdc.noaa.gov/teleconnections/enso/).

The data are explored using statistical software E-View and package 3.2.5 distributed by R-software.

This study described the urban environmental issues that influence of dengue disease in the 
future of urban climatic parameters, on urban activities and to develop adaptive solutions for dengue-adaptation for Karachi city. The methods in this study are based on applying oncological statistical treatment, fractal dimension with rescaled range Hurst exponent for epidemical dengue fever impact of urban climate on the urban morphology. The significant techniques carried out in this research may be classified into following parts. In section 2.1, our mathematical oncological diagnostic treatment methods for the epidemiology of dengue fever.

\subsection{Basic Descriptive Oncological Analysis}

In this section, we present basic Oncological statistical analysis for the vector borne dengue disease with influence of climate parameters. The analysis for this study concludes Oncological descriptive statistic and Fractal dimension by Hurst exponent Rescaled range analysis. The basic statistic is obtained to analyze the relationship between the climatic variables and vector borne dengue epidemics. In firstly, we have conduct to auto correlation analysis for the better judgment of the dengue epidemic and climatic parameters, Auto correlation. The ACF is used for the ensuing two persistence, to detect non-randomness data. If data is not random, then identify the appropriate time series techniques, also to check the linearity of the time series data $X_{t}$.

$$
\begin{array}{r}
\mathrm{ACF}=\aleph(s)=\frac{\operatorname{Cov}\left(X_{t}, X_{t-s}\right)}{\sqrt{\operatorname{Var}\left(X_{t}\right) \operatorname{Var}\left(X_{t-s}\right)}}-1 \leq N(s) \leq 1(1) \text { For } \\
\text { stationary } \operatorname{Var}\left(\mathrm{X}_{\mathrm{t}}\right)=\operatorname{Var}\left(\mathrm{X}_{\mathrm{t}-\mathrm{s}}\right) .
\end{array}
$$

We examine the appropriate probability distribution function (PDF), Normal Distribution is one, resulting in a random interaction of physical parameters studying [42]. Mathematically, is described as a general distribution. Normal distribution formula The
Normal probability distribution function is defined as

$f(x ; \mu, \delta)=\frac{1}{\sqrt{2 \pi} . \sigma} e^{-\frac{1}{2 \sigma^{2}}(x-\mu)^{2}}(2)$

Where, mean $(\mu)$ and standard deviation $(\sigma)$ of data set.

\subsection{Fractal Rescaled Range Method in}

\section{Dengue Epidemics}

This second section intended to examine the dengue epidemics. The main concerned of Fractal Dimension with Hurst Exponent Rescaled range method is of enormous consequence for the human disease [38]. Fractal dimensions are normally assessment of various numbers that are related to fractals [4, $25,36,40]$. In urban analysis, Batty, Longley and Xie were study fractal dimensions of planar urban form and urban dynamics $[5,6$, $7,14]$. Box-counting or box dimension is one of the most widely employed dimensions $[25,30]$. The box dimension of urban form ranges from 0 to 2. Conversely, the box dimension range of urban fractal dimension (D) is $1<D<2[5,7,25$, 28]. Although, these studies have presented some exciting hypothetical formulations and observed results revealing the fractal nature of urban form and growth, they are not systematic in the sense that cities were not selected according to a spatial scheme (e.g. city or population size hierarchy) and an ordinary set of parameters (i.e. map coverage, resolution, scale) The fractal dimension $D$, can be state mathematically as

$$
B(p, r)=\{x: \operatorname{dist}(x, p)<r\}
$$

Where, $B(p, r)$ is Hausdorff dimension with radius $r$ and center $p$, where dist. $(x, p)$ is the distance between point $x$ and point suppose $x$ be a subset of Rn covered by $N(r)$ open covers of radius reduced or else equal to $r$. 
When $r$ tends to $0, N(r)$ increments is approximately $1 / \mathrm{r}$ conferring to power,

$$
D=\lim _{r \rightarrow 0}\left(-\frac{\log N(r)}{\log r}\right)
$$

Where $D$ is called Hausdorff dimension meanwhile, $\log r \rightarrow \infty$ the negative sign is necessary so that $D$ is positive)

$$
N(r) \approx \text { constr }^{D}
$$

$N(r)$ is increments of asymptotically by $r^{D}$.

$$
D=\frac{\log \left[\frac{N_{n-1}}{N_{n}}\right]}{\log \left[\frac{r_{n}}{r_{n-1}}\right]}
$$

Where $N_{n}$ is the number of units required to navigate a curve with a scale ${ }^{r}$. There exist other more simplified dimensions such as capability of dimension (entropies and exploiting), Renyi and correlation dimension [ 7 , $23,43,48$ ]. Similarly, self-affinity (self-affine) may be stated as several power law e.g. Hurst exponent $\boldsymbol{H}$. These powers are possibly nonintegers and collectively called as Fractal dimensions as several of these can be associated by a quantity of law e.g. $H=2-D$

The Hurst exponent can even be applied in urban environmental studies, where it is used to population models. It is usually used as a measure of the fractal (geometric) scaling in the data series, providing an assess for shortand long-term memory of a time series of significance and has widely applicability for time series analysis [25, 41, 42, 48]. The Hurst exponent is commonly calculated for the test of obtained data sets to describe noisy data set. It is also utilized in differentiate stochastic processes. Hurst exponent series between 0 and 1, a time series can be classified into three categories. If $\mathrm{H}=0.5$ then it shows a random series (Brownian process) is a self-affine fractal. If $0<\mathrm{H}<0.5$ then it designates an antipersistent series (random walk) can be considered as having $\mathrm{H}=0$. In case of $0.5<\mathrm{H}<$ 1 it indicates a persistent series [42].

The Rescaled Range Analysis (R/S) algorithm is one of the most commonly used methods for estimation of exponent estimates. The R / S algorithms are estimated that the value of the Hurst exponent is based on an empirical data for the range of dependency based data set 33-35. In this technique, the ratio of two main variables, the range of time series data (the maximum value is subtracted by minimum value) and the standard deviation is known as Hurst exponent [42]. If there is a trend exists, Hurst exponent can deduce an imminent value. It has initiated that time series and Hurst exponent are correlated. It is created by a dynamic system that has a strong statistically significant degree when a segment is automatically interrupted by a correlated signal. The rescaled range $R / S$ is defined as the average of a given sequence of observation $\left(X_{t}\right)$ as

$$
\begin{gathered}
\langle\mathrm{X}\rangle_{\mathrm{t}}=\frac{1}{\tau} \sum_{\mathrm{t}=0}^{\tau-1} \quad \mathrm{X}_{\mathrm{t}} \\
\mathrm{Y}=(t, \tau)=\sum_{t=0}^{\tau-1}\left[X_{t}-\langle X\rangle_{t}\right] \\
\mathrm{R}(\tau)=\max _{0 \leq t \leq \tau-1} Y(t, \tau)-\min _{0 \leq t \leq \tau-1} Y(t, \tau) \\
\mathrm{S}(\tau)=\left(\frac{1}{\tau} \sum_{t=0}^{\tau-1}\left(X_{t}-\langle X\rangle_{t}\right)^{2}\right)^{\frac{1}{2}} \\
\mathrm{R} / \mathrm{S}(\tau)=\frac{R(\tau)}{S(\tau)}
\end{gathered}
$$

We can now apply

$$
\frac{\mathrm{R}}{\mathrm{S}} \propto \tau^{\mathrm{H}}
$$


Where $\mathrm{H}$ is the Hurst exponent.

\subsection{Normality Test for Epidemiological Dengue Disease}

In our case study, we have selected parametric normality test for verification of dengue epidemic seasonal months and all over data intervals are linear or nonlinear by using the Anderson-Darling test. To assess the nature of the distribution of the data (histogram with probability density function) and the deviation from normality using Kolmogorov-Smirnov test and Anderson-Darling test.

The Kolmogorov-Smirnov test is based on the Empirical distribution function (ECDF).

Given $N$ ordered points $x_{1} \ldots \ldots . x_{n}$. The ECDF is defined as:

$$
E_{n=\frac{n(i)}{N}}(9)
$$

Where, $n(i)$ is the number of points less than $x_{i}$ and the $x_{i}$ are ordered from the smallest to the largest value. The Kolmogorov-Smirnov statistic

is defined as.D $\max _{0 \leq i \leq N}\left|F\left(x_{i}\right)-\frac{n(i)}{N}\right|$

Where, $\mathrm{F}$ is the theoretical cumulative distribution of the continuous distribution to be tested. In case the distribution is normal, the null hypothesis is rejected if the test statistic $D$ is greater than the critical value obtained from the table. The value of Probability $>D$ is the $p$ value. The null hypothesis that the data are normally distributed is rejected $[29,45]$. The ADT differs from the K-S test on the influence given to the tails of the distributions. In fact, it was designed to detect discrepancies in the tails [10, $32,43]$. All though the ADT is similar to the Kolmogorov-Smirnov test, but it gives more insight information related to the tails of the distribution. The strength of this test is that it does not depend on the number of intervals. The Anderson-Darling test can only be used for the input data sample which is a weakness of the test [26, 45]. We apply the ADT [AndersonDarling test], where the hypothesis testing is based on comparing the parameter in question with the large values of the statistic [1, 29, 44]. Anderson-Darling test statistic is defined

$$
A^{2}=N-S(11)
$$

Where,

$$
\mathrm{S}=\sum_{i=1}^{N} \frac{(2 i-1)}{N}\left[\ln F\left(x_{i}\right)+\left(1-F\left(x_{N+1-i}\right)\right)\right](12)
$$

Here, $F$ is the cumulative distribution function of the distribution being tested and xiare ordered. The test is one sided and we reject the null hypothesis if the value of $A$ is greater than the critical value. Also, the value of Probability > A 2 is the $p$-value. If the $p$-value is less than the level of significance a then the null hypothesis is rejected. The Anderson-Darling test are specifically intending to identify inconsistency in the tails of the distribution [24, $29,45]$.

\section{RESULTS AND DISCUSSIONS}

The research strategy overdue this study is to give consideration in the complex dynamics of vector borne dengue infectious epidemic with respect to climatic influence over to the city of Karachi region of Pakistan. We have chosen the basis statistical oncological analysis to examine the linear and non-linear dependency and non-stationarity of data by applying the $A R(p)$ correlation ACF residuals plots before calculating Fractal Dimension with Hurst Exponent (Rescaled Range) analysis. Now here, firstly we initiated brief narrative of the monthly and yearly dengue epidemic with dynamical change of climatic parameters by statistical test from January 2001 to December 2020. The yearly time variation of dengue epidemics in the city of Karachi has displayed in Fig. 1.3. It has shown a vibrant disparity for the yearly, the maximum dengue fever counts 6043 
occurred in 2013, indicated enormous number of dengue epidemic transmission and seasonal pattern is displayed in Fig.1.4 that exposed the seasonal peak for outbreaks of dengue from August to November (table1).

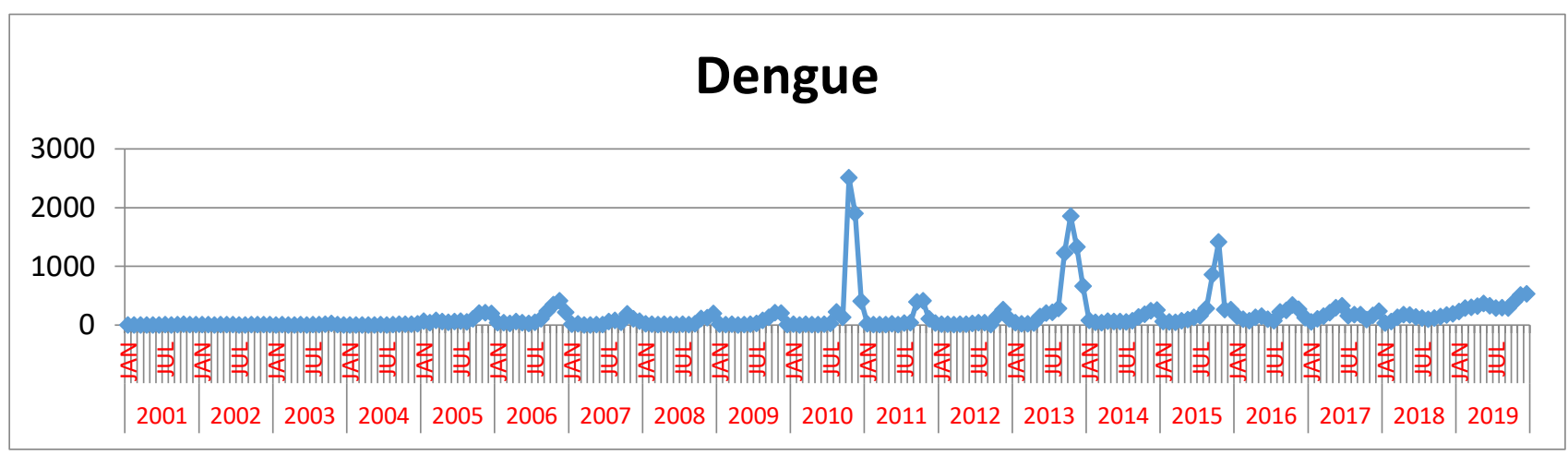

Figure 1.3. The monthly time series plot of dengue epidemic at Karachi region from 2001 to 2020.

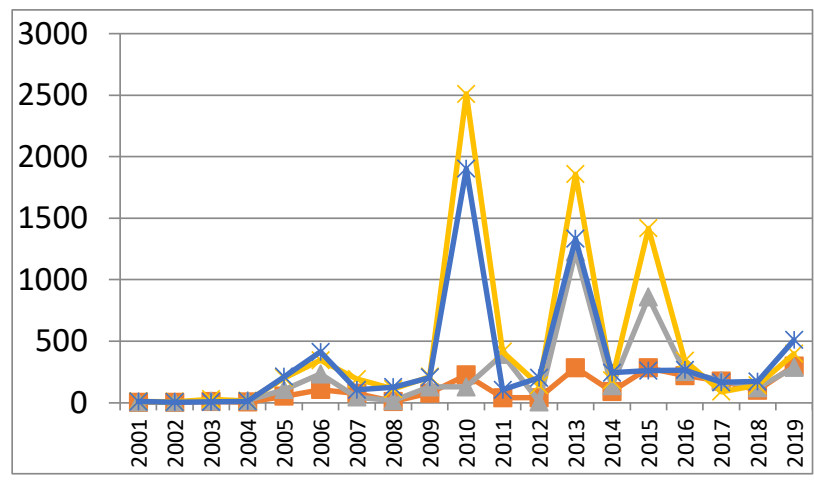

Figure 1.4. The monthly seasonal (August to November) time series pattern of Dengue epidemic at Karachi region from 2001 to 2020.

The autocorrelation described as the correlation among the unbiased data points. This technique is established as lag function in equation (1) related to dengue and temperature, dengue and ENSO time series are depicted in Figure 1.5. It indicates that the climatic data set has linear robust short range depends clearly on the series of time series (figure 1.5. correlation function of dengue incidence and climatic parameters indicate the 95\% C.I (figure 1.5 (a)). The temperature plot indicated delay positive correlation with dengue epidemic (figure 1.5 (b), ENSO index plot exhibits negative associate with dengue epidemic (figure 1.5(c)).

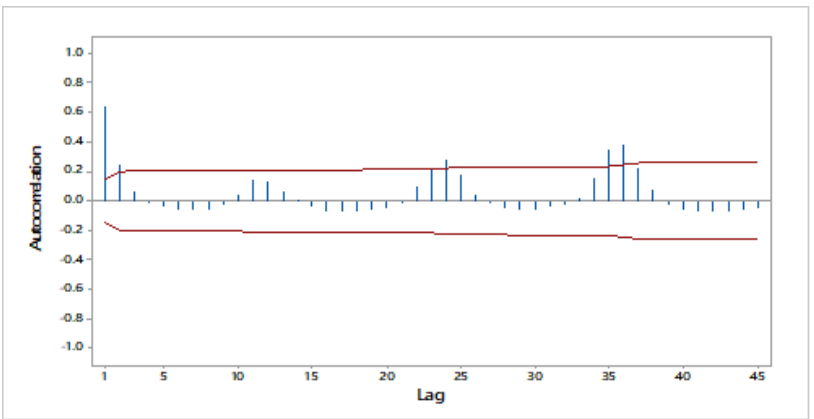

Figure 1.5 (a). The monthly time series plot of cross autocorrelation function (5\% significance limit) of dengue epidemic data at Karachi region from 2001 to 2020 .

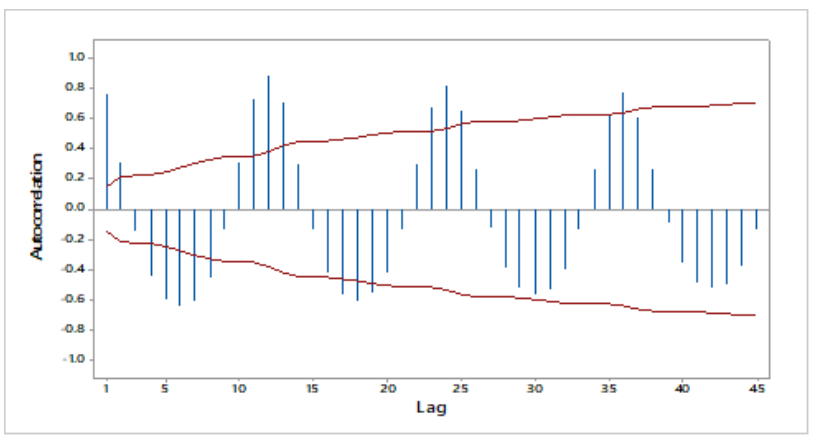

Figure 1.5. (b). The monthly time series plot of cross autocorrelation function (5\% significance limit) of mean Temperature $\left({ }^{\circ} \mathrm{C}\right)$ data at Karachi region from 2001 to 2020. 


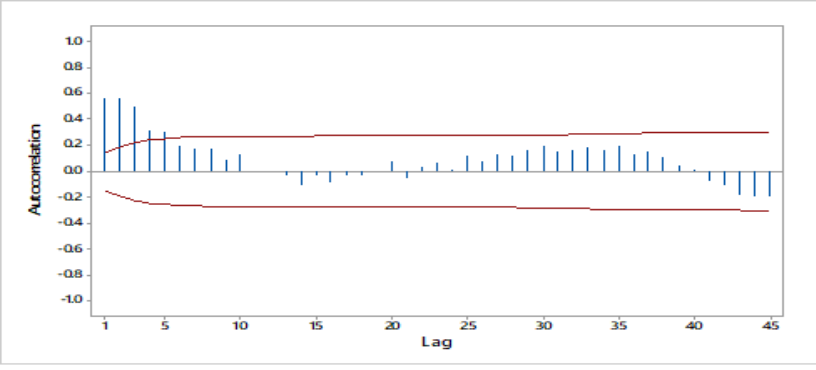

Figure 1.5. (c). The monthly time series plot of cross autocorrelation function (5\% significance limit) of ENSO 3.4 index data at Karachi region from 2001 to 2020.

We have chosen two statistical approaches, which are classified into two factions, descriptive statistical test and hypothesis acquired technique, and these methods are helpful to statistical analysis / Oncological treatments of the time series data. thus, test of the skewness, kurtosis and standard deviation values are judged as descriptive test while other approach consist the normality test such as ADT and KST, as well as the preceding test for check the level stationary of the time series data, test name is Augmented DickeyFuller (ADF) critical statistical test for different level $(1 \%, 5 \%$ and $10 \%)$.

From table 1, peak seasonal and total months(yearly) oncological statistical results summary are described that the skewness coefficient value for dengue and ENSO is positive except land temperature is negative skewness, the positive skewness for dengue epidemic indicated the right skew, irregularity and deviation of the normal distribution, Kurtosis exemplify the peaked affirmed a quite smooth distribution and positive value of kurtosis confirmed a peaked distribution in exhibited in fig 1.6, we examine that the histogram for normal distribution is positive skewed to the right which is depiction of total dengue epidemic. Total yearly and peak seasonal month high dengue incidences with Temperature and ENSO index are detected in the months of September, October and November depicts in figure 1.6.

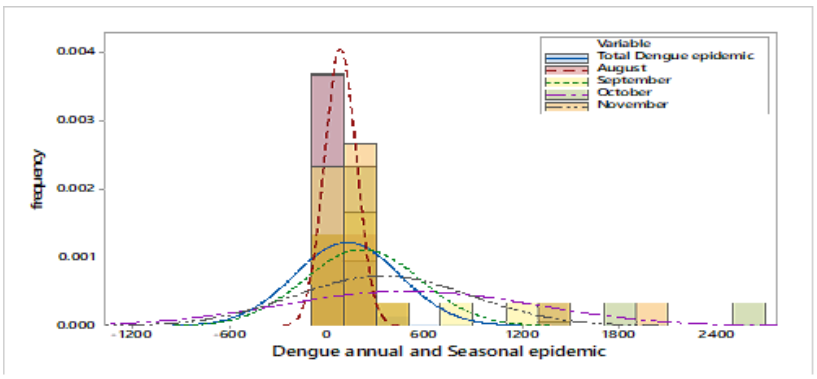

Figure 1.6. (a). The monthly seasonal plots of histograms of dengue for (a) august (b) September (c) October (d) November and (e) Total months for 2001 to 2020.

Table1. Summary basic statistics total monthly and seasonal months for dengue epidemic with Temperature and ENSO 3.4 Index for 2001 to 2020.

\begin{tabular}{|c|c|c|c|c|c|c|}
\hline Statistics & Parameters & August & September & October & November & Total Months \\
\hline \multirow[t]{3}{*}{ Mean } & Dengue & 85.5 & 92.1 & 503 & 346 & 127.3 \\
\hline & Temp & 29.642 & 29.876 & 29.435 & 25.595 & 27.273 \\
\hline & ENSO & -0.18 & 0.22 & 0.423 & 0.293 & 0.279 \\
\hline \multirow[t]{3}{*}{ Minimum } & Dengue & 3 & 4 & 8 & 5 & 0 \\
\hline & Temp & 28.639 & Minimum & 28.116 & 23.74 & 16.39 \\
\hline & ENSO & -2.4 & -2.7 & -2.8 & -1.5 & -5.2 \\
\hline \multirow[t]{3}{*}{ Maximum } & Dengue & 284 & 1225 & 2510 & 1902 & 2510 \\
\hline & Temp & 30.406 & 30.73 & 30.555 & 26.673 & 33.79 \\
\hline & ENSO & 3 & 3.7 & 2.9 & 2.2 & 4.8 \\
\hline Median & Dengue & 52 & 107 & 193 & 204 & 26.5 \\
\hline
\end{tabular}




\begin{tabular}{|c|c|c|c|c|c|c|}
\hline & Temp & 29.645 & 30.16 & 29.5 & 25.64 & 29.211 \\
\hline & ENSO & -0.3 & 0.3 & -0.1 & 0.1 & 0.1 \\
\hline \multirow[t]{3}{*}{ Range } & Dengue & 281 & 1221 & 2502 & 1897 & 2510 \\
\hline & Temp & 1.768 & 2.663 & 2.439 & 2.933 & 17.4 \\
\hline & ENSO & 5.4 & 6.4 & 5.7 & 3.7 & 10 \\
\hline \multirow[t]{3}{*}{ IQR } & Dengue & 97 & 221 & 386 & 250 & 97 \\
\hline & Temp & 0.803 & 0.973 & 1.332 & 1.11 & 6.612 \\
\hline & ENSO & 1.9 & 1.6 & 2.2 & 2.2 & 2.175 \\
\hline \multirow[t]{3}{*}{ St. Deviation } & Dengue & 98.4 & 356.5 & 774 & 450 & 326.6 \\
\hline & Temp & 0.535 & 0.748 & 0.785 & 0.738 & 4.403 \\
\hline & ENSO & 1.374 & 1.516 & 1.637 & 1.251 & 1.566 \\
\hline \multirow[t]{3}{*}{ Skewness } & Dengue & 1.33 & 2.2 & 1.89 & 2.37 & 4.87 \\
\hline & Temp & -0.54 & -1.09 & -0.22 & -0.93 & -0.74 \\
\hline & ENSO & 0.69 & 0.45 & -0.07 & 0.22 & 0.37 \\
\hline \multirow[t]{3}{*}{ Kurtosis } & Dengue & 0.52 & 4.36 & 2.54 & 5.09 & 26.33 \\
\hline & Temp & -0.18 & 0.89 & -1.09 & 1.51 & -0.72 \\
\hline & ENSO & 0.88 & 1.29 & -0.58 & -1.46 & 0.61 \\
\hline \multirow[t]{3}{*}{ KS-statistic } & Dengue & 0.264 & 0.327 & 0.364 & 0.362 & 0.348 \\
\hline & Temp & 0.166 & 0.181 & 0.108 & 0.149 & 0.19 \\
\hline & ENSO & 0.135 & 0.201 & 0.124 & 1.149 & 1.081 \\
\hline
\end{tabular}

Before applying the fractal, range scaled $R / S$ analysis, we have checked the Dickey-fuller unit root test for dengue epidemic with climatic parameters temperature and ENSO for stationarity at level $(1 \%, 5 \%$ and $10 \%)$, are depicted in table 1.2. The $\mathrm{p}$ - value of all of three datasets was less than $0.05(p<0.05)$, these results are shown that the level statistic value is greater than critical values, time series indicated stationary. In our situation, we have selected ADT and KST to find the normality ofdengue epidemic peak seasonal in monthly data series.

Table 1.2(a). Summary of the ADF test statistic for different significance fordengue epidemic

\begin{tabular}{lccc} 
& & t-Statistic & Prob. $^{*}$ \\
\hline Augmented & Dickey- & & \\
Fuller test statistic & -7.275562 & 0.0000
\end{tabular}

Test critical

values

$$
\begin{array}{ll}
1 \% \text { level } & -4.007084 \\
5 \% \text { level } & -3.433651
\end{array}
$$

$-3.140697$

$10 \%$ level

Table 1.2(b). Summary of the ADF test statistic for different significance for Temperature $\left({ }^{\circ} \mathrm{C}\right)$

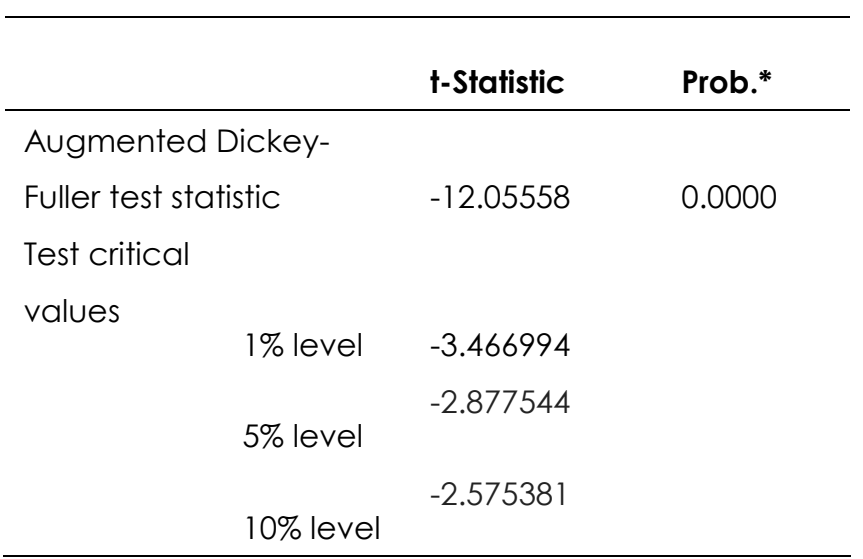


Table 1.2(c).Summary of the ADF test statistic for different significance forENSO

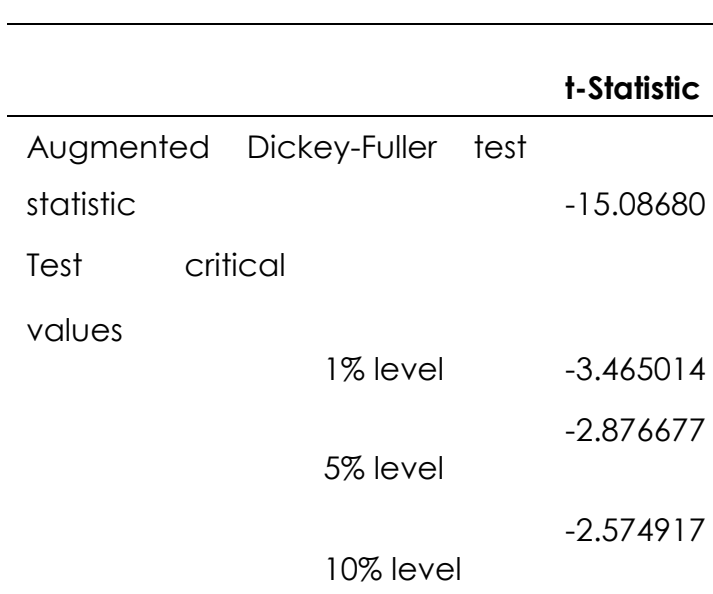

\subsection{Analysis of Self Affine Fractal Dimension} $(R / S)$ Consequences

This section, first we have determined complexity of the vector-borne dengue fever transmission by using the persistency analysis with Rescaled range method. As well, Hurst Exponent are assessed by $A R(1)$ correlation residual of the entire monthly dengue epidemic with climatic parameters land temperature and ENSO to remove the linearity of the data set, we obtain the total annual dengue outbreaks and the months, which has dengue transmission frequency $\geq 12$ in 16 years as detailed in $\S$ 2.1.Therefore, we have total annual dengue disease occurrence, and the occurrences of the peak seasonal months of the year (September, October and November) are enchanting for applying analysis on. After applying the trend analysis method, we get some appropriate results ( $a=0.05$ level), which has helpful for further persistency scrutinizing, the total annual trend equation $y(t)=65.81 \mathrm{D}$ 30.327 and seasonal peaked months august is $y(t)=15.015 D-33.825$, September trend is $y(t)=$ 40.438D-120.6, October is $y(t)=71.379 D-107.23$, and November is $y(t)=38.15 D+17.1$. Based on our oncological statistical treatment, we are getting that the slope line of total annual trend is become the highest, correspondingly, months of September and October are indicated more frequent months for dengue epidemic. consequently, the output of normal probability distribution function(pdf) of dengue total months shown peak seasonal months data series exciting, indicated that month of September to November did not untilled normal distribution (figure 1.7).Exceeding, the land surface temperature and ENSO normal probability histogram of the total annual months and seasonal months (August to November) exposed an increasing right tail appropriate distribution(figure $1.6(\mathrm{~b}-\mathrm{c})$ ). The positive kurtosis value for dengue seasonal months are suggested an increasing nature in fig 1.6 (c), for normal distribution histogram. It is suggested that the role of climatic influence is also relevant for dengue transmission.

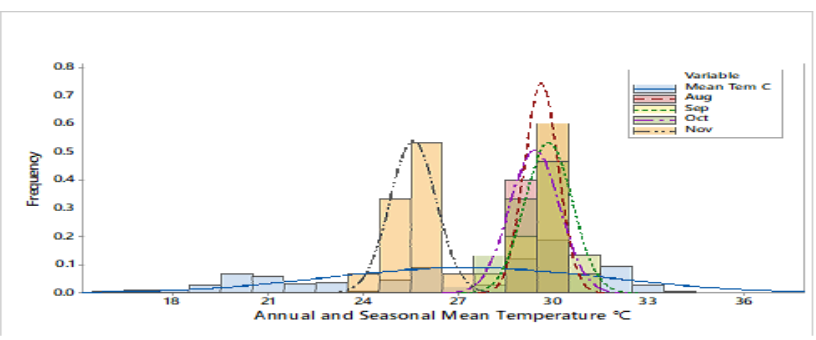

Figure 1.6.(b). The monthly seasonal plots of histograms of Mean Temperature $\left({ }^{\circ} \mathrm{C}\right.$ ) for (a) august (b) September (c) October (d) November and (e) Total months for 2001 to 2020.

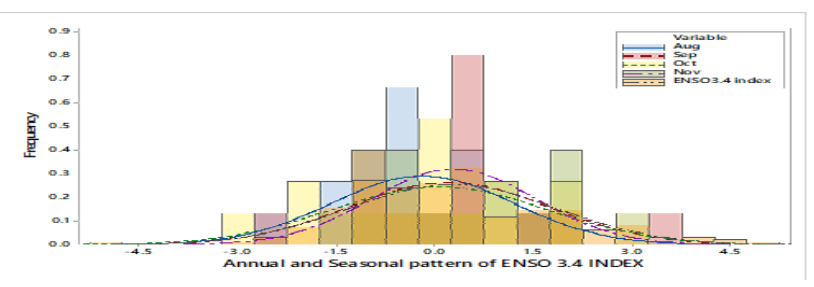

Figure 1.6.(c). The monthly seasonal plots of histograms of ENSO for (a) august (b) September (c) October (d) November and (e) Total months for 2001 to 2020 . 


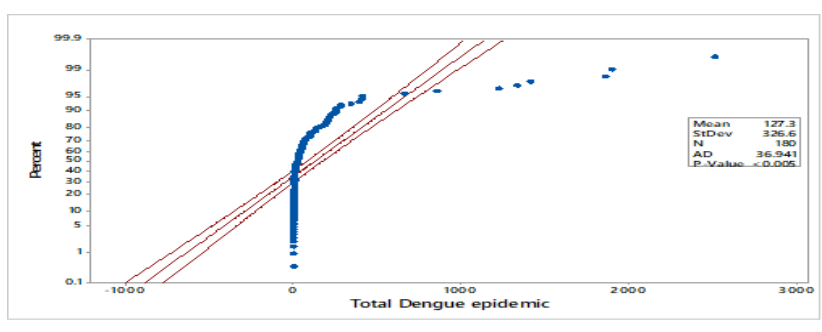

Figure 1.7. The monthly time series Plot of fitted distribution normal probability density function (pdf) of Dengue epidemic from 2001 to 2020.

To further investigation the fluctuating behavior of total annual and month's dengue fever epidemic, the estimated values of the fractal dimension has been tabulated in table 1.3.

Table 1.3.The detail procedure of rescaled range analysis and Fractal dimension for dengue epidemic, climate temperature and ENSO 3.4 index over Karachi region to estimate Hurst for year 2001 to 2020.

\begin{tabular}{|c|c|c|c|c|c|c|c|}
\hline Months & & $R / S$ & $\log ^{2}(R / S)$ & D & H & Nature & Correlation \\
\hline \multirow[t]{3}{*}{ Jan } & Dengue & $2.824 \lcm{092}$ & 1.4977 & 1.467 & 0.533 & Persistence & Positive \\
\hline & ENSO & 3.405797 & 1.7679 & 1.022 & 0.978 & Persistence & Positive \\
\hline & Temp & 3.612069 & 1.8528 & 1.013 & 0.987 & Persistence & Positive \\
\hline \multirow[t]{3}{*}{ Feb } & Dengue & 3.694836 & 1.8855 & 1.474 & 0.526 & Persistence & Positive \\
\hline & ENSO & 4.052468 & 2.0188 & 1.018 & 0.982 & Persistence & Positive \\
\hline & Temp & 4.434032 & 2.1486 & 1.029 & 0.971 & Persistence & Positive \\
\hline \multirow[t]{3}{*}{ Mar } & Dengue & 4.763689 & 2.252 & 1.481 & 0.519 & Persistence & Positive \\
\hline & ENSO & 3.457946 & 1.7899 & 1.044 & 0.956 & Persistence & Positive \\
\hline & Temp & 4.145946 & 2.0517 & 1.058 & 0.942 & Persistence & Positive \\
\hline \multirow[t]{3}{*}{ April } & Dengue & 3.962779 & 1.9865 & 1.437 & 0.503 & Persistence & Positive \\
\hline & ENSO & 4.183058 & 2.0645 & 1.041 & 0.959 & Persistence & Positive \\
\hline & Temp & 3.948609 & 1.9813 & 1.023 & 0.977 & Persistence & Positive \\
\hline \multirow[t]{3}{*}{ May } & Dengue & 2.442341 & 1.2882 & 1.419 & 0.581 & Persistence & Positive \\
\hline & ENSO & 3.164955 & 1.6621 & 1.008 & 0.992 & Persistence & Positive \\
\hline & Temp & 4.27065 & 2.0944 & 1.011 & 0.989 & Persistence & Positive \\
\hline \multirow[t]{3}{*}{ Jun } & Dengue & 2.90625 & 1.5391 & 1.488 & 0.512 & Persistence & Positive \\
\hline & ENSO & 3.994066 & 1.9978 & 1.01 & 0.99 & Persistence & Positive \\
\hline & Temp & 4.085614 & 2.0305 & 1.007 & 0.993 & Persistence & Positive \\
\hline \multirow[t]{3}{*}{ Jul } & Dengue & 8.433962 & 3.0762 & 1.788 & 0.212 & Anti-Persistence & Negative \\
\hline & ENSO & 4.178971 & 2.0631 & 1.039 & 0.961 & Persistence & Positive \\
\hline & Temp & 4.305465 & 2.1061 & 1.027 & 0.973 & Persistence & Positive \\
\hline \multirow[t]{3}{*}{ Aug } & Dengue & 1.911208 & 0.9344 & 1.313 & 0.687 & Persistence & Positive \\
\hline & ENSO & 3.926016 & 1.973 & 1.045 & 0.955 & Persistence & Positive \\
\hline & Temp & 3.347506 & 1.743 & 1.002 & 0.998 & Persistence & Positive \\
\hline \multirow[t]{3}{*}{ Sep } & Dengue & 9.869565 & 3.3029 & 1.816 & 0.184 & Anti-Persistence & Negative \\
\hline & ENSO & 4.38557 & 2.1327 & 1.056 & 0.944 & Persistence & Positive \\
\hline & Temp & 3.507872 & 1.8105 & 1.018 & 0.982 & Persistence & Positive \\
\hline \multirow[t]{2}{*}{ Oct } & Dengue & 3.494382 & 1.805 & 1.555 & 0.445 & Anti-Persistence & Negative \\
\hline & ENSO & 3.670295 & 1.8758 & 1.027 & 0.973 & Persistence & Positive \\
\hline
\end{tabular}




\begin{tabular}{cccccccc}
\multirow{4}{*}{ Nov } & Temp & 3.785166 & 1.9203 & 1.018 & 0.982 & Persistence & Positive \\
& Dengue & 15.94915 & 3.9954 & 1.882 & 0.118 & Anti-Persistence & Negative \\
& ENSO & 2.964487 & 1.5677 & 1.037 & 0.963 & Persistence & Positive \\
\multirow{4}{*}{ Dec } & Temp & 4.245527 & 2.0859 & 1.007 & 0.993 & Persistence & Positive \\
& Dengue & 32.89831 & 5.0399 & 1.941 & 0.059 & Anti-Persistence & Negative \\
& ENSO & 3.285036 & 1.7159 & 1.057 & 0.943 & Persistence & Positive \\
\multirow{3}{*}{ Total } & Temp & 4.822924 & 2.2699 & 1.014 & 0.986 & Persistence & Positive \\
& Dengue & 7.938716 & 2.9889 & 1.184 & 0.816 & Persistence & Positive \\
& ENSO & 6.402647 & 2.6786 & 1 & 1 & Persistence & Positive \\
& Temp & 3.961964 & 1.9862 & 1.001 & 0.999 & Persistence & Positive \\
\hline
\end{tabular}

The dengue epidemic fractal dimension results are shown the trend of anti-persistency in the months of September to December, nonetheless the total annual(months) for dengue, Temperature and ENSO time series data sets are shown persistent (increasing positive trend correlation), the fractal actions of the seasonal pattern of dengue epidemic with respect to climate land surface temperature and ENSO index (months of September to November) indicated the anti-persistency (negative correlated) peak months for dengue

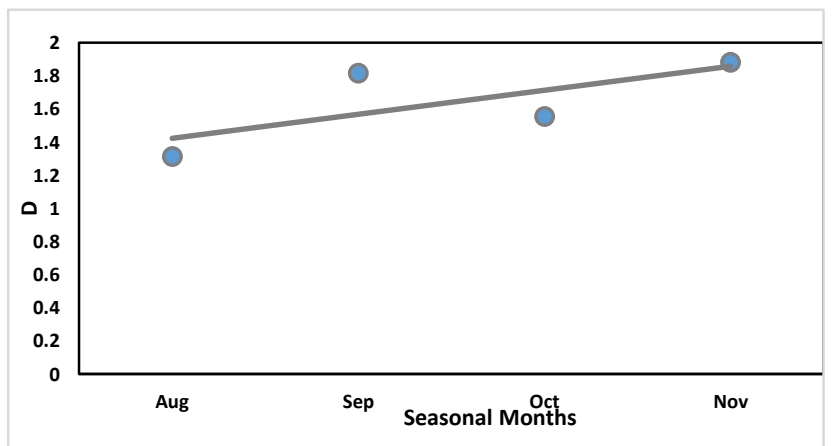

epidemic data series, climate parameters time series for every months are shown persistency, all over climatic time series are increasing trend and positive correlated. The slope of the appropriate seasonal pattern (September to November) for dengue epidemic and climate parameters fitted lines calculated log R/S verse seasonal months as specify table 1.3. The Fractal dimension $D$ of dengue epidemic for annual and peak months values are displayed in figure 1.8 and 1.9 .

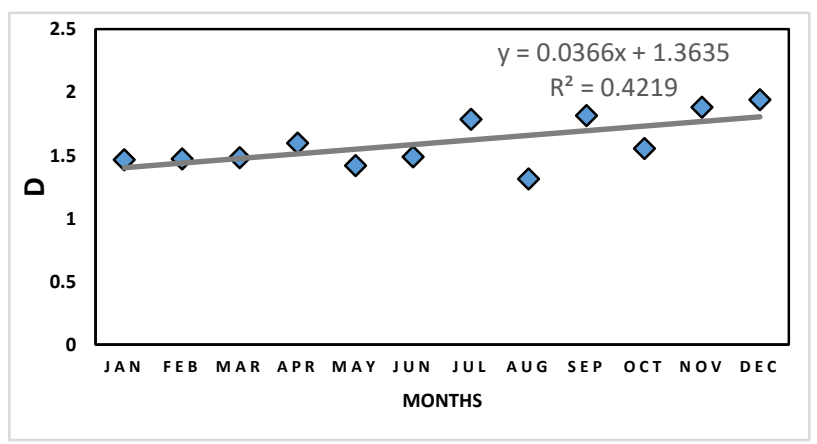

Figure 1.8. The time series plot of Fractal $R / S$ analysis, the least square procedure for total annual dengue epidemic for Karachi region where the slope of fitted line estimates the Hurst Exponent for the year 2001 to 2020.

Figure 1.9. The time series plot of Fractal $R / S$ analysis, the least square procedure for Seasonal Peak monthly dengue epidemic for Karachi region where the slope of fitted line estimates the Hurst Exponent for the year 2001 to 2020.

The Hurst exponent attained for individual months including seasonal peak months for dengue epidemic thru Temperature and ENSO index time series during the month of January to December (from 2001 to 2020).The results for self-affine fractal dimension values correlated to Hurst exponent values exposed in table 1.3, dengue transition seasonal peak months for September to November indicated anti- 
persistence of time series for all other months except December and July which confirmed persistent, temperature and ENSO index all months are shown persistent as the fractal dimension value $D<1.5$ and the value of $H$ is greater than 0.5, Moreover, we have selected Anderson-Darling test that associates whether epidemiological dengue disease seasonal months data is nonlinear or linear, evidence found here has confirmed the nonlinearity and anti-persistency behavior of dengue peak season (September to November)monthly data time series that reduces the linearity from dengue series data, the ADT statistics values for the month of September is $A=2.156$, October is $A=2.351$ and November is $A=2.365$ (null of hypothesis $\mathrm{HO}$ rejected), we have concluded that the peak season of the dengue epidemics is detached the linearity because the non-linearity are occurred.

\section{CONCLUSION}

In this study we have performed to time series study to investigate the epidemiological aspects by using mathematical oncological treatment, also conclude influence of climatic variability on dengue transmission in the city of Karachi for eighteen years monthly (from 2001 to 2020) time series data. In the urban environment of greater Puntarenas, it was possible to discern relationships between dengue incidence and local weather data. By the intention of investigation of the linear and nonlinear variations in the dengue epidemic, basic oncological statistical analysis and Fractal dimension related to the range scaled dimension Hurst exponent methodology, are found that the scarce seasonal jagged months September to November for high arboviral dengue disease of humans and robust linear interdependence obviously survive in seasonal epidemic of dengue in Karachi region.

Moreover, this research/statement strength contain that fractal analysis to study the epidemiological changing aspects of vectorborne dengue diseases with climate parameters (land surface temperature and ENSO index). The results achieved that entirely total months and seasonal peak months on climatic parameters (Temperature and ENSO index) attentiveness strong persistency behavior (positive correlation), Hurst Exponent values is very closed to 1.Meanwhile peak seasonal data set for dengue epidemics shown anti-persistent behavior (negative correlation) excluding the august, further months January, February, April, May and June that revealed persistence i.e. positive correlation, Our $R / S$ results has highlighted that R/S Fractal analysis was more appropriate for humans health disease (dengue fever) evolution through temperate and ENSO to recognize the fractal nature of data sets. Finally, the normality statistical test for epidemiological treatment of dengue disease also inveterate the non-linear performance for seasonal months of dengue influence at Karachi region. The normality test is found that the linearity for peak season is rejected (null of hypothesis) through the ADT. Definitely, the results allowed that the dengue transmission in seasonal periods should be rejected on the basis of non-linear dependence and evidence suggested that the non-linear inherent structure is contemporary in epidemic dengue data, the unbiased of this research are in the fractal nature of it. To identify Karachi's area of stability. In the future, this research will be extended to include dengue outbreaks in urban morphological structure, in which our developed urban population density map for sixteen towns (7-Districts) will be more 
helpful for individual investigate the dengue influence in different towns of city .In addition, the new multivariate analysis approach will be employed to increase the perception of data analysis, thus providing scientists more efficiently to the urban environment in the Karachi region.

\section{ACKNOWLEDGMENT}

We are thankful to the Dengue Survival Cells, Karachi metrological department and National Oceanic and Atmospheric (www.ncdc.noad.gov/teleconnections/enso/) for providing Dengue fever outbreaks data, climatic and weather data. The content is the part of first author's Doctoral thesis to be submitted Mathematical Sciences Research Center Federal Urdu University Karachi.

\section{DECLARATIONS}

\section{Conflicts of interest/Competing}

interests:There is no conflict of interest.

Authors' contributions:Shaheen Abbas and Muhammad llyas conceptualization, methodology and reviewing, Muhammad llyas and Afzal Ali data collection and Analysis, Shaheen Abbas,

Muhammad llyas and Afzal Ali write up the paper and proof reading by Sadaqat Hussain and Syed

\section{Akhter Raza.}

\section{REFERENCES}

[1] A. Rizk, M. Simaan, W. D. Halpin and J. R Wilson, Fitting beta distributions based on sample data. J Constr Eng Manag 120(1994) 288-305.

[2] Ahmed, S. Afrozuddin, J. S. Siddiqi, S. Quaiser and A. Ahmed Siddiqui, Analysis of Climatic Structure with Karachi Dengue Outbreak. J Basic Appl Sci 1 1:(2015)544-552.

[3] Atique, Suleman, Shabbir Syed Abdul, ChienYeh Hsu, and Ting-Wu Chuang. Meteorological influences on dengue transmission in Pakistan. Asian Pac J Trop Med 9(2016) 954-61.
[4] B. Andrew, R. E. Green and M. Jenkins, Measuring the changing state of nature. Trends Ecol Evol 18:(2003)326-330.

[5] B. Michael and P. A. Longley, Fractal cities: a geometry of form and function. (Academic press), (1994).

[6] B. Michael and P. A. Longley, Fractal-based description of urban form. Environ Plann B Plann Des 14(1987) 123-134.

[7] B. Michael and P. A. Longley, Urban shapes as fractals. Area (1987b) 215-22.

[8] Balmaseda, Angel et al, Assessment of the World Health Organization scheme for classification of dengue severity in Nicaragua. Am J Trop Med Hyg 73(2005) 1059-1062.

[9] C. Antonietta, ENSO diversity in the NCAR CCSM4 climate model. J Geophys Res Oceans 118 (2013) 4755-4770.

[10] D' Agostino Ralph B, Goodness-of-fittechniques. (CRC press), (1986).

[11] D. L. Engels, Chitsulo, A. Montresor, and L Savioli, The global epidemiological situation of schistosomiasis and new approaches to control and research. Acta tropica 82(2002) 139-146.

[12] Ebi, L. Kristie and J. Nealon, Dengue in a changing climate. Environmental research, 151 (2016) 115-123.

[13] Focks, D Ae, and Dave D Chadee, Pupal survey: an epidemiologically significant surveillance method for Aedes aegypti: an example using data from Trinidad', Am J Trop Med Hyg 56(1997) 159-167.

[14] Garmendia, Alfonso, and Adela Salvador, Fractal dimension of birds population sizes time series. Math Biosci 206(2007) 155-171.

[15] Gibbons, Robert V, and David W Vaughn, Dengue: an escalating problem. Bmj 324(2002) 1563-1566.

[16] Gill, CA, The Influence of Humidity on the Life History of Mosquitoes and on their Power to Transmit Infection. Trans R Soc Trop Med Hyg 14(1921). 
[17] Gill, CA, The Relationship of Malaria \& Rainfall', Indian J Med Res, 7(1920).

[18] Gill, Clifford Allchin. The Rôle oï Meteorology in Malaria. Indian J Med Res 8 (1921).

[19] Glattre, Eystein, Jan F Nygård, and Eystein Skjerve, Fractal epidemiology. Epidemiology 20 (2009) 468.

[20] Goldberger, Ary L, and Bruce J West. Applications of Nonlinear Dynamics to Clinical Cardiology a. Ann N Y Acad Sci 504(1987) 195-213. [21] Gubler, DJ, G Kuno, GE Sather, M Velez, and ANDA Oliver. Mosquito cell cultures and specific monoclonal antibodies in surveillance for dengue viruses. The Am J Trop Med Hyg 33(1984) 158-165.

[22] Gubler, Duane J, Epidemic dengue and dengue hemorrhagic fever: a global public health problem in the 21 st century. In. emerging infections 1 (Am Soci of Micro), (1998).

[23] Hassan D, Abbas S, Ansari M. R. K and Jan B. The study of Sunspots and K-index data in the perspective of probability distributions. Int J Phys and SociSci 4(2014) 23.

[24] Holcomb, Edward Warren. Estimating parameters of stable distributions with application to nonlife insurance. Unpublished Ph. D. dissertation, University of Tennessee, (1973).

[25] Hoskin, Paul WO. 'Patterns of chaos: fractal statistics and the oscillatory chemistry of zircon', Geochimica et Cosmochimica Acta, 64(2000) 1905-23.

[26] Hussain MA, S Abbas and MRK Ansari. Persistency analysis of cyclone history in Arabian sea. The Nucleus 48(2011) 273-277.

[27] Ilyas, M., Abbas, S., Naz, S. A., \& Abbas, M. The impact of climatic influence on dengue infectious disease in Karachi Pakistan. Int J of Mosquito Res, 6 (2019) 04-13.

[28] Inchausti, Pablo and John Halley, Investigating long-term ecological variability using the global population dynamics database. Science 293(2001) 655-57.
[29] Kale, Malhar Dilip and Ferry Butar Butar, Fractal analysis of time series and distribution properties of Hurst exponent. Sam Houston State University, (2005).

[30] Katsev, Sergei, and Ivan L'Heureux, Are Hurst exponents estimated from short or irregular time series meaningful? Computers \& Geosciences 29 (2003) 1085-1089.

[31] Kuno Goro, Review of the factors modulating dengue transmission. Epidemiol Rev 17(1995) 321 35.

[32] Law, Averill M, W David Kelton and W David Kelton. Simulation modeling and analysis. In.: McGraw-Hill New York, (1991).

[33] Le, Thanh Hoa, Measuring urban morphology for adaptation to climate change in Ho Chi Minh City, (2014).

[34] Maio, C, and C Schexnayder, Simulation model probability distribution functions: Relationships between input data and goodnessof-fit tests. In IAARC/IFAC/IEEE. International symposium (1999) 103-108.

[35] Mandelbrot, Benoit B. The fractal geometry of nature (WH freeman New York), (1982).

[36] Meakin, Paul, Fractals, scaling and growth far from equilibrium (Cambridge university press), (1998).

[37] Mena, Nelson, Adriana Troyo, Roger BonillaCarrión, and Ólger Calderón-Arguedas. Factores asociados con la incidencia de dengue en Costa Rica. Revista Panamericana de Salud Publica. 29 (2011) 234-242.

[38] Nygård, Jan F, and Eystein Glattre, Fractal analysis of time series in epidemiology: Is there information hidden in the noise? Norsk Epidemiologi 13 (2003).

[39] Rusch, Hannah L, and Jim Perry, Dengue and the Landscape: A Threat to Public Health. National Center for Case Study Teaching In Science. 1-4 (2011). 
[40] S. Abbas and M. R. K. Ansari, Pattern of Karachi Katchi-Abadies. Journal J Basic Appl Sci. 6(2) (2010a).

[41] S. Abbas Shaheen and M. R. K. Ansari, Urbanization of Karachi Different Stages of Population Growth. J Basic Appl Sci 6(2) (2010b).

[42] S. Abbas Urban Dynamics In The Perspective Of Environmental Change: Karachi as a Case Study (Doctoral dissertation, Federal Urdu University of Arts, Sciences and Technology Gulshan-e-labal Campus Karachi (Pakistan)). (2011)

[43] Shen, Guoqiang, Fractal dimension and fractal growth of urbanized areas, Int J Geogr Inf Sci, 16 (2002) 419-437.

[44] Shpilberg, David C, 'The probability distribution of fire loss amount. J Risk Insur: (1977) 103-115.
[45] Thode, Henry C. Testing for normality (CRC press), (2002).

[46] Troyo, Adriana, Douglas O Fuller, Olger Calderón-Arguedas, Mayra E Solano, and John C Beier. Urban structure and dengue incidence in Puntarenas, Costa Rica. Singap J Trop Geogr 30(2009) 265-282.

[47] Weaver, Scott C, and Nikos Vasilakis, Molecular evolution of dengue viruses: contributions of phylogenetics to understanding the history and epidemiology of the preeminent arboviral disease. Infection, genetics and evolution 9 (2009) 523-540.

[48] Wiens, Brian L, when log-normal and gamma models give different results: a case study. Am Stat, $53(1999) 89-93$

Received: 31 Aug. 2021. Revised/Accepted: 05 Dec. 2021. 